\title{
References
}

World Health Organization. Global Tuberculosis Report 2013. Geneva, WHO, 2013.

Weyer K, Mirzayev F, Migliori GB, et al. Rapid molecular TB diagnosis: evidence, policy making and global implementation of Xpert MTB/RIF. Eur Respir J 2013; 42: 252-271.

3 Denkinger CM, Nicolau I, Ramsay A, et al. Are peripheral microscopy centres ready for next generation molecular tuberculosis diagnostics? Eur Respir J 2013; 42: 544-547.

UNITAID. Tuberculosis: Diagnostics Technology and Market Landscape 2013. 2nd Edn. Genenva, WHO, 2013. Graham SM, Ahmed T, Amanullah F, et al. Evaluation of tuberculosis diagnostics in children: 1. Proposed clinical case definitions for classification of intrathoracic tuberculosis disease. Consensus from an expert panel. J Infect Dis 2012; 205: Suppl. 2, S199-S208.

6 Batz H-G, Cooke GS, Reid SD. Towards lab-free tuberculosis diagnosis. Lausanne, Médecins Sans Frontières, 2011.

7 Denkinger CM, Kik SV, Pai M. Robust, reliable and resilient: designing molecular tuberculosis tests for microscopy centers in developing countries. Expert Rev Mol Diagn 2013; 13: 763-767.

8 Wells WA, Boehme CC, Cobelens FG, et al. Alignment of new tuberculosis drug regimens and drug susceptibility testing: a framework for action. Lancet Infect Dis 2013; 13: 449-458.

9 Pai M, Denkinger CM, Kik SV, et al. Gamma Interferon release assays for detection of Mycobacterium tuberculosis Infection. Clin Microbiol Rev 2014; 27: 3-20.

10 Wallis RS, Kim P, Cole S, et al. Tuberculosis biomarkers discovery: developments, needs, and challenges. Lancet Infect Dis 2013; 13: 362-372.

11 Kik SV, Denkinger CM, Chedore P, et al. Replacing smear microscopy for the diagnosis of tuberculosis: what is the market potential? Eur Respir J 2014; 43: 1793-1796.

12 Pai NP, Vadnais C, Denkinger C, et al. Point-of-care testing for infectious diseases: diversity, complexity, and barriers in low- and middle-income countries. PLoS Med 2012; 9: e1001306.

13 World Health Organization. An international roadmap for tuberculosis research: towards a world free of tuberculosis. Geneva, WHO, 2011.

14 World Health Organization. Priorities for tuberculosis research. A report of the disease reference group on TB, leprosy and Buruli ulcer. Geneva, WHO, 2013.

\section{6-year follow-up of 522 HIV-positive individuals screened for Mycobacterium tuberculosis infection in Denmark}

\section{To the Editor:}

We report the result of a 6-year follow-up study among 522 HIV-positive patients showing a positive predictive value (PPV) of 7\% (two out of 28) and a negative predictive value (NPV) of 100\% (478 out of 478) for developing active tuberculosis (TB) using the QuantiFERON-TB Gold In-Tube test (QFT-IT).

Denmark is a low TB incidence country with an annual incidence rate of six cases per 100000 persons and a HIV prevalence of 70 cases per 100000 persons [1]. Among HIV-positive patients registered in the Danish HIV Cohort, comprising all known HIV-positive individuals in Denmark, the incidence rate of TB was as high as 8.2 cases per 1000 person-years [2]. The rate was highest before and within the first 6 months of antiretroviral treatment (ART) and among patients with known TB risk factors.

HIV is a well-known risk factor for developing active TB, increasing the risk $>20$-fold compared to HIVuninfected individuals [3-5]. In Eastern Europe the risk of death in HIV patients co-infected with TB is especially high [6].

Isoniazid preventive treatment (IPT) has shown to be effective in preventing reactivation of TB in HIV positive individuals [7-9], and the treatment has been recommended by the World Health Organization and the US Centers for Disease Control and Prevention [10].

In order to prevent further spread of the disease and prevent infection, IPT of HIV-positive individuals is now recommended in high-burden regions, independently of tuberculin skin test or interferon-gamma release assay results [10]. 
In Denmark, screening and treatment of latent TB infection is only recommended in individuals with recent TB exposure and candidates of tumour necrosis factor (TNF) $-\alpha$ inhibitor treatment, but no specific guidelines exist for HIV-positive individuals.

The objective of this study was to estimate the predictive value of the QFT-IT among HIV-positive individuals in Denmark. The study is a follow-up study of a cohort of 590 HIV-positive individuals screened for latent TB infection in 2004-2005 [11].

We conducted a prospective cohort study at the outpatient clinic at Copenhagen University Hospital (Hvidovre, Denmark) to determine the risk of developing active TB. In the outpatient clinic we followed 1122 HIV-positive individuals who attended three to four annual visits. QFT-IT screening was routinely offered in the period between December 2004 and May 2005, and the result of this screening has been reported previously [11]. Initially, $590 \mathrm{HIV}$-positive individuals were screened with the QFT-IT test. Information on patient status (still attending the clinic, dead or lost to follow-up) at December 31, 2010 was obtained for each patient via the hospital patient registry using the Civil Registration System (CRS). Patients referred to other hospitals in Denmark but who were still alive were included. Information on TB diagnosis was derived from the Danish National TB register.

TB is a mandatory notifiable disease in Denmark and all incident cases diagnosed by a medical doctor are recorded in the Danish National TB register. The CRS provides all citizens in Denmark with a unique personal identifier, which can be used to trace a person through all public registries. For the present study all incident TB cases recorded among the study participants were retrieved from the Danish National TB register between the date of recruitment and December 31, 2010. Additional information on ethnicity, death or emigration was obtained from the CRS. Data including sex, ethnic origin, HIV status and CD4 cell counts for all $1122 \mathrm{HIV}$-positive individuals seen in the outpatient clinic were derived from the Danish HIV Cohort [12] and the local hospital registry. HIV-positive patients were grouped into Caucasian and nonCaucasian, where persons born in Africa, Asia or Latin America were defined as non-Caucasian.

Between 2004 and 2005, patients were tested with the QFT-IT as previously described [11], and all QFT-ITpositive individuals were screened for signs and symptoms of active TB. None of the QFT-IT-positive patients were offered chemoprophylactic TB treatment. One patient, who developed active TB within 30 days of the QFT-IT test, was excluded from the analysis because he was considered to have active TB at the time of testing. The study was notified to the Danish Data Protection Agency (Jr.nr.2005-41-5520).

Out of $1122 \mathrm{HIV}$-positive individuals registered in the outpatient clinic, 590 (52\%) accepted QFT-IT testing. 68 patients were either lost to follow-up due to emigration or died during the study period and were excluded from analysis. None of the 68 patients had been registered with TB in the Danish National TB register during the time after screening. Thus, complete follow-up data for 522 (88.5\%) of the screened HIV patients were available.

The study participants were representative of the patients seen in our outpatient clinic with regards to sex, ethnicity, HIV status, CD4 count and ART (table 1). In the study cohort of 522 individuals, 412 (79\%) were

TABLE 1 Baseline characteristics for the study population and the total HIV population seen in the outpatient clinic

QFT-IT screened HIV positive

\begin{tabular}{lcc}
\hline Subjects $n$ & 522 & 1122 \\
Males & $422(80)$ & $812(72)$ \\
HIV status & $123(22)$ & $230(15)$ \\
$\quad$ AIDS & $435(83)$ & $858(76)$ \\
$\quad$ ART & 531 & 499 \\
CD4 cell count & $9(2)$ & $19(3)$ \\
$0-99$ cells $\mu \mathrm{L}^{-1}$ & $25(5)$ & $37(6)$ \\
$100-199$ cells $\mu \mathrm{L}^{-1}$ & $48(9)$ & $69(10)$ \\
$200-300$ cells $\mu \mathrm{L}^{-1}$ & $434(84)$ & $546(81)$ \\
$>300$ cells $\mu \mathrm{L}^{-1}$ & $412(79)$ & $878(78)$ \\
Caucasian & $110(21)$ & $244(22)$ \\
\hline Non-Caucasian &
\end{tabular}

Data are presented as $\mathrm{n}(\%)$ or mean, unless otherwise stated. There was no statistically significant difference between the two groups. QFT-IT: Quantiferon-TB Gold In-Tube; ART: antiretroviral treatment. \#: defined as persons born in Africa, Asia or Latin America. 
Caucasian and $110(21 \%)$ were non-Caucasian. This distribution is comparable to all 1122 HIV individuals seen in our outpatient clinic where 878 (78\%) were Caucasian and $244(22 \%)$ were non-Caucasian.

Out of the 522 individuals with complete follow-up data, 28 (5\%) were QFT-IT positive, 478 (91\%) were negative and $16(4 \%)$ were indeterminate. Among the 506 individuals with a valid QFT response the QFT was positive in $13.3 \%$ (14 out of 105) of the non-Caucasians compared to 3.5\% (14 out of 401) of the Caucasians resulting in a three to five times higher odds of a positive QFT-IT test result among nonCaucasians. We found a low proportion of indeterminate results, which could be explained by the fact that $90 \%$ of the patients had CD4 cell count $>200$ cells $\cdot \mu \mathrm{L}^{-1}$ and $\sim 80 \%$ were receiving ART.

During the 6 years of follow-up, two $(7 \%)$ cases of TB were seen among the 28 patients with a positive QFT-IT test. The TB cases were diagnosed 11 and 54 months after QFT-IT testing and both TB patients were male. One patient was of non-Caucasian origin (the Philippines), he had a CD4 cell count of 301 cells $\cdot \mu \mathrm{L}^{-1}$ and no other risk factors. The second patient was of Danish origin with ongoing alcohol and intravenous drug abuse and a CD4 cell count of $530 \mathrm{cells} \cdot \mu \mathrm{L}^{-1}$. No cases of TB were reported among the 478 QFT-IT-negative patients or among the 16 patients with indeterminate results.

Thus, among the HIV-positive individuals without active TB at the time of screening and with available data at follow-up, the NPV of the QFT-IT was 100\% (478 out of 478; 95\% CI 99-100) and the PPV was 7\% (two out of $28 ; 95 \%$ CI 1-22).

In this study, the number needed to treat with isoniazid to potentially avoid one TB case was 14 (two out of 28) and the number needed to test to identify one QFT-IT-positive individual was 18.6 (28 out of 522).

The strength of this study is the completeness of the data. We had follow-up results for $88.5 \%$ of the initial cohort and all patients were followed for a minimum of 6 years. Thus, due to the use of register information, the risk of information bias was minimal. The CRS allows for follow-up of study participants even if they leave the clinic, which also limits loss to follow-up. Furthermore, the personal identifier assures that any TB diagnosis among participants will be registered. The comparability of the study population with the total population of HIV patients in our outpatient clinic minimises the risk of participation bias and may allow us to extrapolate our result to all our patients (table 1).

One limitation is the low number of TB cases during the follow-up period, which limits the possibility of calculating measures of association based on TB as outcome parameter. Furthermore, data on larger cohorts should be collected in order to determine the PPV in HIV-positive individuals with low CD4 cell counts.

Although the number of TB cases in this study was small, our finding of a PPV of $7 \%$ is similar to that reported from Austria where AicheLburg et al. [13] found a PPV of 8.7\%. We can conclude that the QFT-IT test with a NPV of a 100\% can be considered a safe test for ruling out risk of TB among immunocompetent HIV-positive individuals in a low endemic country like Denmark. Based on our study and others, there is an increased risk of progressing to TB in QFT-IT-positive HIV-positive individuals that should not be ignored [9].

The evidence to recommend IPT to HIV-positive patients is overwhelming even in the presence of effective antiretroviral treatment [8-10]. This combined effect is shown in high-burden countries, whereas data from low-endemic regions are limited. Being born in a TB high-endemic region is a risk factor for latent TB infection and for developing active TB among HIV-positive individuals in Denmark [2, 11] and other European countries [7]. It is a matter of debate whether screening should be limited to high-risk groups in low-endemic regions. In our study, one of the two cases of active TB was seen in a Danish born male; therefore, we suggest that all HIV-positive individuals should be considered for screening irrespective of the pre-test probability of infection. Testing HIV-positive individuals should be performed with the intension to treat them with IPT.

The cost of one QFT-IT test compared to the total cost of complete HIV care in Denmark is minimal and a discussion of the guidelines is recommended.

In conclusion, the present study shows that QFT-IT screening could be safely used for screening immunocompetent HIV-positive individuals in Denmark to prevent future TB in this group.

@ERSpublications

The QFT-IT test can be safely used in HIV-positive patients; consider isoniazid prophylaxis if QFTIT positive http://ow.ly/vBFOP

Christian Soborg ${ }^{1}$, Morten Ruhwald ${ }^{1,2}$, Peter H. Andersen ${ }^{2}$ and Pernille Ravn ${ }^{3}$

${ }^{1}$ Dept of Infectious Disease, Copenhagen University Hospital, Hvidovre, Denmark. ${ }^{2}$ Statens Serum Institut, Copenhagen, Denmark. ${ }^{3}$ Dept of Infectious Disease, Nordsjælland Hospital, Hillerød, Denmark. 
Correspondence: Christian Soborg, Dept of Infectious Diseases, Hvidovre Hospital, Kettegårds alle 30, Hvidovre 2650, Denmark. E-mail: borg1@dadlnet.dk

Received: Sept 302013 | Accepted after revision: April 032014 | First published online: May 22014

Support statement: Copenhagen University Hospital (Hvidovre, Denmark) hold patents on the use of IP-10 as a marker for infection with Mycobacterium tuberculosis. P. Ravn and M. Ruhwald are registered as co-inventors. P.H. Andersen is employed by Statens Serum Institute which holds patent rights on antigens used in the QFT-IT test.

Conflict of interest: Disclosures can be found alongside the online version of this article at erj.ersjournals.com

Acknowledgements: We are grateful to Christina Thorstenisson (Dept of Infectious Disease, Copenhagen University Hospital, Hvidovre, Denmark) for statistical assistance and Bolette Søborg (Dept of Epidemiology Research, Statens Serum Institut, Copenhagen, Denmark) for enthusiastic commenting in the manuscript.

\title{
References
}

1 EPI-NEWS. Tuberculosis 2011, Part I. No. 50, 2012. www.ssi.dk/English/News/EPI-NEWS/2012/No\%2050\% 20-\%202012.aspx Date last updated: December 12, 2012. Date last accessed: January 3, 2014.

2 Taarnhoj GA, Engsig FN, Ravn P, et al. Incidence, risk factors and mortality of tuberculosis in Danish HIV patients 1995-2007. BMC Pulm Med 2011; 11: 26.

3 Horsburgh CR Jr. Priorities for the treatment of latent tuberculosis infection in the United States. N Engl J Med 2004; 350: 2060-2067.

4 Lawn SD, Bekker LG, Wood R. How effectively does HAART restore immune responses to Mycobacterium tuberculosis? Implications for tuberculosis control. AIDS 2005; 19: 1113-1124.

5 Pontali E, Pasticci MB, Matteelli A, et al. Tuberculosis and HIV co-infection: do we have a surveillance system in Europe? Eur Respir J 2011; 38: 1258-1260.

6 Podlekareva DN, Panteleev AM, Grint D, et al. Short- and long-term mortality and causes of death in HIV/ tuberculosis patients in Europe. Eur Respir J 2014; 43: 166-177.

7 Elzi L, Schlegel M, Weber R, et al. Reducing tuberculosis incidence by tuberculin skin testing, preventive treatment, and antiretroviral therapy in an area of low tuberculosis transmission. Clin Infect Dis 2007; 44: 94-102.

8 Whalen CC, Johnson JL, Okwera A, et al. A trial of three regimens to prevent tuberculosis in Ugandan adults infected with the human immunodeficiency virus. Uganda-Case Western Reserve University Research Collaboration. N Engl J Med 1997; 337: 801-808.

9 Durovni B, Saraceni V, Moulton LH, et al. Effect of improved tuberculosis screening and isoniazid preventive therapy on incidence of tuberculosis and death in patients with HIV in clinics in Rio de Janeiro, Brazil: a stepped wedge, cluster-randomised trial. Lancet Infect Dis 2013; 13: 852-858.

10 World Health Organisation. WHO policy on collaborative TB/HIV activities: guidelines for national programmes and other stake holders. Geneva, WHO, 2012.

11 Brock I, Ruhwald M, Lundgren B, et al. Latent tuberculosis in HIV positive, diagnosed by the M. tuberculosis specific interferon-gamma test. Respir Res 2006; 7: 56.

12 The Danish HIV Cohort Study. www.rigshospitalet.dk/RHenglish/Menu/Departments+and+Clinics/Finsen+Centre/ Department+of+Infectious+Diseases_old/Research/ Date last updated: November 13, 2013. Date last accessed: January 3, 2014.

13 Aichelburg MC, Rieger A, Breitenecker F, et al. Detection and prediction of active tuberculosis disease by a wholeblood interferon-gamma release assay in HIV-1-infected individuals. Clin Infect Dis 2009; 48: 954-962.

\section{Tuberculosis elimination: dream or reality? The case of Cyprus}

\author{
To the Editor:
}

The World Health Organization (WHO)-recommended strategy of tuberculosis (TB) control has evolved from the DOTS (directly observed treatment, short course) strategy to the Stop TB strategy [1, 2]. By breaking the chain of transmission through rapid diagnosis and effective treatment of infectious TB cases, this strategy allowed the development of national TB programmes and proved to be highly cost-effective $[3,4]$.

However, in order to accelerate the decline of the TB pandemic, WHO has planned a post-2015 strategy focusing on the concept of TB elimination [5-8]. To reach elimination (defined as fewer than one sputum smear-positive case per million inhabitants), countries should ensure that diagnosis and treatment of latent TB infection (LTBI) supplements detection and treatment of active TB disease [7-10].

A recent survey conducted by the European Respiratory Society (as part of its 2012-2013 Presidential plan) and WHO in Europe has demonstrated that the majority of countries do not have all the interventions 